\title{
Explicitly solvable systems of two autonomous first-order ordinary differential equations with homogeneous quadratic right-hand sides
}

\author{
Francesco Calogero $^{a, b, 1}$ and Farrin Payandeh ${ }^{c, 2}$ \\ a Physics Department, University of Rome "La Sapienza", Rome, Italy \\ ${ }^{b}$ Istituto Nazionale di Fisica Nucleare, Sezione di Roma 1 \\ ${ }^{c}$ Department of Physics, Payame Noor University (PNU) \\ PO Box 19395-3697 Tehran, Iran \\ 1 francesco.calogero@uniroma1.it; francesco.calogero@roma1.infn.it \\ 2 f_payandeh@pnu.ac.ir; farrinpayandeh@yahoo.com
}

Received Date: 8 April 2021; Accepted Date: 15 May 2021

\begin{abstract}
After tersely reviewing the various meanings that can be given to the property of a system of nonlinear ODEs to be solvable, we identify a special case of the system of two first-order ODEs with homogeneous quadratic right-hand sides which is explicitly solvable. It is identified by 2 explicit algebraic constraints on the 6 a priori arbitrary parameters that characterize this system. Simple extensions of this model to cases with nonhomogeneous quadratic right-hand sides are also identified, including isochronous cases.
\end{abstract}

\section{Introduction}

In this paper we mainly focus on the following system of 2 first-order ODEs with homogeneous quadratic right-hand sides:

$$
\dot{x}_{n}(t)=c_{n 1}\left[x_{1}(t)\right]^{2}+c_{n 2} x_{1}(t) x_{2}(t)+c_{n 3}\left[x_{2}(t)\right]^{2}, \quad n=1,2 .
$$

Notation 1-1. Above and hereafter $t$ is the independent variable, and superimposed dots indicate differentiation with respect to $t$. The 2 functions $x_{n}(t)$ are the dependent variables, and other dependent variables $y_{n}(t)$ are introduced below. Often below the dependence of these variables on $t$ shall not be explicitly displayed, when this omission is unlikely to cause any misunderstanding. The 6 (t-independent) parameters $c_{n \ell}$ are a priori arbitrary, but a posteriori we shall identify 2 constraints on their values; and other $t$-independent parameters - such as $a_{n m}, b_{n m}$, etc.- shall be introduced below. All

(C) The author(s). Distributed under a Creative Commons Attribution 4.0 International License 
variables and parameters can be complex numbers (but of course the subcase in which they are real numbers is of special interest in applicative contexts); we shall instead generally think of the independent variable $t$ as time, but analytic continuation to complex values of $t$ - and of other analogous time-like variables such as $\tau$, see below - shall also be discussed. Generally each of the 2 indices $n$ and $m$ take the 2 values 1 and 2 , and the index $\ell$ the 3 values $1,2,3$.

The system (11) is a prototypical system of nonlinearly-coupled ODEs and as such has over time been studied in many theoretical investigations and also utilized in an enormous number of applicative contexts; a much too large research universe to make it possible to mention all relevant references. Here we limit ourselves to quote the path-breaking papers by René Garnier [5], and the very recent papers [2] and [3], whose topics are quite close to those treated in the present paper, as discussed in the last two Sections $\mathbf{6}$ and $\mathbf{7}$, where possible future developments are also tersely outlined; and just one textbook reference [4] (of course the interested reader can trace additional references from those quoted in these sources).

The main finding of the present paper is the identification (see Sections 2, 3 and 4) of a subclass of the model (11) - characterized by 2 explicit algebraic constraints on the 6 coefficients $c_{n \ell}$ (see below the 2 eqs. (36) ) - which then allows the explicit solution of the initial-values problem for this system (1), as detailed in Proposition 2-2.

Invariance properties of the system (1) and some simplifications of it are reported in Section 5.

Some extensions of the model (11) to analogous systems with non-homogeneous quadratic right-hand sides - including isochronous versions - are discussed in Section 6.

A comparison with previous findings, and a very terse mention of possible future developments, are provided in Section 7.

Let us complete this introductory Section 1 with a terse review-complementing the analogous treatment provided in [2] of the various meanings that can be given to the property of a system of nonlinear ODEs to be solvable, and more specifically to be explicitly solvable.

As already noted in [2], the statement that a system of nonlinear ODEs - such as (11) - is solvable by quadratures is somewhat misleading, when it only implies that the independent variable $t$ can be identified as a function of an appropriate combination of the dependent variables represented by an integral which cannot be explicitly performed or that can be expressed as a named function - such as, say, a hypergeometric functionwhich cannot be readily inverted. A less unsatisfactory outcome is when that function is a polynomial, implying that its inversion yields an algebraic function, since this has significant implications, especially in terms of the analytic structure of the solution when considered as a function of complex $t$; although of course a generic polynomial cannot be explicitly inverted - i. e., its roots identified - unless its degree does not exceed 4.

In the present paper the statement that a system of nonlinearly-coupled ODEs is explicitly solvable indicates that the solution of the corresponding initial-values problem can be exhibited as an elementary function of the independent variable $t$, involving parameters themselves expressed, in terms of the original parameters of the model, by explicit formulas only involving elementary functions; the final formulas expressing the parameters of the solution being nevertheless, possibly, quite complicated, being produced by a finite (generally short) chain of explicit relations applied sequentially (see examples below). 


\section{Main results}

The following 2 Propositions are proven in the following Section 3.

Proposition 2-1. The explicit solution of the initial-values problem for the system

$$
\begin{aligned}
& \dot{y}_{1}(t)=\left[y_{1}(t)\right]^{2}, \\
& \dot{y}_{2}(t)=\rho_{1}\left[y_{1}(t)\right]^{2}+\rho_{2} y_{1}(t) y_{2}(t)+\left[y_{2}(t)\right]^{2},
\end{aligned}
$$

where $\rho_{1}$ and $\rho_{2}$ are 2 arbitrary parameters, reads as follows:

$$
\begin{aligned}
& y_{1}(t)=y_{1}(0)\left[1-y_{1}(0) t\right]^{-1} \\
& y_{2}(t)=y_{1}(0)\left[1-y_{1}(0) t\right]^{-1} u(t) \\
& u(t)=\frac{u_{+}\left[u(0)-u_{-}\right]-u_{-}\left[u(0)-u_{+}\right]\left[1-y_{1}(0) t\right]^{-\Delta}}{u(0)-u_{-}-\left[u(0)-u_{+}\right]\left[1-y_{1}(0) t\right]^{-\Delta}}, \\
& u(0)=y_{2}(0) / y_{1}(0), \quad u_{ \pm}=\left(1-\rho_{2} \pm \Delta\right) / 2 \\
& \Delta=\sqrt{\left(1-\rho_{2}\right)^{2}-4 \rho_{1}} .
\end{aligned}
$$

This solution is valid for arbitrary initial data $y_{1}(0)$ and $y_{2}(0)$, provided $y_{1}(0) \neq 0$. If instead $y_{1}(0)=0$ implying $y_{1}(t)=y_{1}(0)=0$-in which case some of the formulas (3) become undetermined - then of course $y_{2}(t)=y_{2}(0)\left[1-y_{2}(0) t\right]^{-1}$ (see (2b) with $\left.y_{1}(t)=0\right)$.

Remark 2-1. Note that this solution is clearly invariant under the assignment of the sign of $\Delta$ (not defined by eq. (3e $)$ ): see $(\sqrt{3 \mathrm{c}})$ and the definition $(\underline{3 \mathrm{~d}})$ of the 2 parameters $u_{ \pm}$.

Proposition 2-2. The initial-values problem - with generic initial data-for the system (1) is explicitly solvable provided the 6 a priori arbitrary parameters $c_{n \ell}(n=1,2$; $\ell=1,2,3)$ are expressed in terms of the $6=2+4$ a priori arbitrary parameters $\rho_{1}, \rho_{2}$ and $a_{n m}$ or $b_{n m}(n=1,2 ; m=1,2)$ by the following formulas:

$$
\begin{aligned}
& c_{n 1}=b_{n 1}\left(a_{11}\right)^{2}+b_{n 2}\left[\rho_{1}\left(a_{11}\right)^{2}+\left(\rho_{2} a_{11}+a_{21}\right) a_{21}\right], n=1,2, \\
& c_{n 2}=2 b_{n 1} a_{11} a_{12}+b_{n 2}\left[2 \rho_{1} a_{11} a_{12}+\rho_{2}\left(a_{11} a_{22}+a_{12} a_{21}\right)+2 a_{21} a_{22}\right], n=1,2, \\
& c_{n 3}=b_{n 1}\left(a_{12}\right)^{2}+b_{n 2}\left[\rho_{1}\left(a_{12}\right)^{2}+\left(\rho_{2} a_{12}+a_{22}\right) a_{22}\right], n=1,2 .
\end{aligned}
$$

Here the 4 parameters $a_{n m}$ and the 4 parameters $b_{n m}$ are related by the following 4 formulas:

$$
a_{11}=b_{22} / B, a_{12}=-b_{12} / B, a_{21}=-b_{21} / B, a_{22}=b_{11} / B
$$


or, equivalently,

$$
b_{11}=a_{22} / A, \quad b_{12}=-a_{12} / A, \quad b_{21}=-a_{21} / A, \quad b_{22}=a_{11} / A,
$$

where

$$
\begin{aligned}
& A=a_{11} a_{22}-a_{12} a_{21}=B^{-1}, \\
& B=b_{11} b_{22}-b_{12} b_{21}=A^{-1} ;
\end{aligned}
$$

obviously implying the possibility to express - via the formulas (44) with $n=1,2$ - the 6 coefficients $c_{n \ell}$ in terms of the 2 parameters $\rho_{1}, \rho_{2}$ and either the 4 a priori arbitrary parameters $a_{n m}$ or the 4 arbitrary parameters $b_{n m}$.

Then the solution of the initial-values problem for the system (11) is related to the explicit solution (3) of the corresponding initial-values problem for the system (2) (see Proposition 2-1) via the following linear relations:

$$
\begin{aligned}
& y_{1}(t)=a_{11} x_{1}(t)+a_{12} x_{2}(t), \quad y_{2}(t)=a_{21} x_{1}(t)+a_{22} x_{2}(t), \\
& x_{1}(t)=b_{11} y_{1}(t)+b_{12} y_{2}(t), \quad x_{2}(t)=b_{21} y_{1}(t)+b_{22} y_{2}(t),
\end{aligned}
$$

which are easily seen to imply the relations (5).

\section{Proofs}

\subsection{Proof of Proposition 2-1}

In this subsection we provide for completeness a proof of Proposition 2-1, although this finding is rather elementary and by no means new (see for instance [5]).

The fact that (3a) provides the solution of the initial-value problem for the ODE (2a) is plain.

Hereafter we assume $y_{1}(0) \neq 0$.

Then set

$$
y_{2}(t)=u(t) y_{1}(t), \quad u(t)=y_{2}(t) / y_{1}(t)
$$

hence

$$
\dot{u}(t)=\left[\dot{y}_{2}(t)-u(t) \dot{y}_{1}(t)\right] / y_{1}(t)
$$

hence, via (2) and (3a),

$$
\dot{u}(t)=y_{1}(0)\left[1-y_{1}(0) t\right]^{-1}\left\{[u(t)]^{2}+\left(\rho_{2}-1\right) u(t)+\rho_{1}\right\},
$$

and since clearly, via the definition of $u_{ \pm}$(see $(3 \mathrm{~d} d)$ ),

$$
[u(t)]^{2}+\left(\rho_{2}-1\right) u(t)+\rho_{1}=\left[u(t)-u_{+}\right]\left[u(t)-u_{-}\right],
$$


the ODE satisfied by $u(t)$ reads as follows:

$$
\dot{u}(t)\left\{\left[u(t)-u_{+}\right]\left[u(t)-u_{-}\right]\right\}^{-1}=y_{1}(0)\left[1-y_{1}(0) t\right]^{-1}
$$

hence, again via the definition of $u_{ \pm}($see $(\underline{3 d})$ ),

$$
\dot{u}(t)\left\{\left[u(t)-u_{+}\right]^{-1}-\left[u(t)-u_{-}\right]^{-1}\right\}=\Delta y_{1}(0)\left[1-y_{1}(0) t\right]^{-1}
$$

which can be immediately integrated, yielding (3c).

The expression of $y_{2}(t)$ (see (3) ) is thereby validated, completing thereby the proof of Proposition 2-1.

Remark 2.1-1. Of course the expression (3C) of $u(t)$ is valid for generic values of the relevant parameters. For the special initial values $u(0)=u_{ \pm}$it yields the trivial result $u(t)=u(0)$. For the special values of the parameters $\rho_{1}$ and $\rho_{2}$ such that $\rho_{1}=\left(1-\rho_{2}\right)^{2} / 4$ implying $\Delta=0($ see $(\underline{3 \mathrm{e}}))$ and (see $(\underline{3 \mathrm{~d}})$ )

$$
u_{+}=u_{-}=\bar{u}=\left(1-\rho_{2}\right) / 2 \text {, }
$$

the expression (3c) of $u(t)$ is replaced by the following formula (implied by (11) with (12)):

$$
u(t)=\frac{u(0)+\bar{u}[u(0)-\bar{u}] \ln \left[1-y_{1}(0) t\right]}{1+[u(0)-\bar{u}] \ln \left[1-y_{1}(0) t\right]} .
$$

\subsection{Proof of Proposition 2-2}

Let us $t$-differentiate the relations (6b) respectively (6a), getting

$$
\dot{x}_{1}=b_{11} \dot{y}_{1}+b_{12} \dot{y}_{2}, \quad \dot{x}_{2}=b_{21} \dot{y}_{1}+b_{22} \dot{y}_{2},
$$

respectively

$$
\dot{y}_{1}=a_{11} \dot{x}_{1}+a_{12} \dot{x}_{2}, \quad \dot{y}_{2}=a_{21} \dot{x}_{1}+a_{22} \dot{x}_{2} .
$$

Hence, from the first of these 2 pairs of relations, we get, via (2),

$$
\dot{x}_{n}=b_{n 1}\left(y_{1}\right)^{2}+b_{n 2}\left[\rho_{1}\left(y_{1}\right)^{2}+\rho_{2} y_{1} y_{2}+\left(y_{2}\right)^{2}\right], \quad n=1,2 ;
$$

and then, via (6a) and a bit of trivial algebra, the system (11) with the expressions (4) of the coefficients $c_{n \ell}$. Proposition 2-2 is thereby proven.

\section{Inversion of the equations (4) with (5)}

In this Section we discuss the important problem to invert the system of algebraic equations (44) with (5), i. e. to express the 2 parameters $\rho_{1}, \rho_{2}$ and the 4 parameters $a_{n m}$-or, equivalently (see (5) ), the 4 parameters $b_{n m}$-in terms of the 6 parameters $c_{n \ell}$; and we 
find 2 constraints on the 6 parameters $c_{n \ell}$ which are required in order to fulfill this task, hence are necessary for the explicit solvability of the system (1) via Proposition 2.2.

As a first step, let us note that the system of 2 ODEs (14b) implies, via the system (1), the following 2 ODEs:

$$
\begin{aligned}
\dot{y}_{n}=\left(a_{n 1} c_{11}\right. & \left.+a_{n 2} c_{21}\right)\left(x_{1}\right)^{2}+\left(a_{n 1} c_{12}+a_{n 2} c_{22}\right) x_{1} x_{2} \\
& +\left(a_{n 1} c_{13}+a_{n 2} c_{23}\right)\left(x_{2}\right)^{2}, \quad n=1,2,
\end{aligned}
$$

hence, via (6b), the following system of 2 ODEs:

$$
\begin{array}{r}
\dot{y}_{n}=\left(a_{n 1} c_{11}+a_{n 2} c_{21}\right)\left(b_{11} y_{1}+b_{12} y_{2}\right)^{2} \\
+\left(a_{n 1} c_{12}+a_{n 2} c_{22}\right)\left(b_{11} y_{1}+b_{12} y_{2}\right)\left(b_{21} y_{1}+b_{22} y_{2}\right) \\
+\left(a_{n 1} c_{13}+a_{n 2} c_{23}\right)\left(b_{21} y_{1}+b_{22} y_{2}\right)^{2}, \quad n=1,2,
\end{array}
$$

hence

$$
\dot{y}_{n}=\gamma_{n 1}\left(y_{1}\right)^{2}+\gamma_{n 2} y_{1} y_{2}+\gamma_{n 3}\left(y_{2}\right)^{2}, \quad n=1,2
$$

with

$$
\begin{gathered}
\gamma_{n 1}=\left(a_{n 1} c_{11}+a_{n 2} c_{21}\right)\left(b_{11}\right)^{2}+\left(a_{n 1} c_{12}+a_{n 2} c_{22}\right) b_{11} b_{21} \\
+\left(a_{n 1} c_{13}+a_{n 2} c_{23}\right)\left(b_{21}\right)^{2}, \quad n=1,2 \\
\gamma_{n 2}=2\left(a_{n 1} c_{11}+a_{n 2} c_{21}\right) b_{11} b_{12}+\left(a_{n 1} c_{12}+a_{n 2} c_{22}\right)\left(b_{11} b_{22}+b_{12} b_{21}\right) \\
+2\left(a_{n 1} c_{13}+a_{n 2} c_{23}\right) b_{21} b_{22}, \quad n=1,2 \\
\gamma_{n 3}=\left(a_{n 1} c_{11}+a_{n 2} c_{21}\right)\left(b_{12}\right)^{2}+\left(a_{n 1} c_{12}+a_{n 2} c_{22}\right) b_{12} b_{22} \\
+\left(a_{n 1} c_{13}+a_{n 2} c_{23}\right)\left(b_{22}\right)^{2}, \quad n=1,2 .
\end{gathered}
$$

And now a comparison of this system of ODEs with the system (2) implies

$$
\gamma_{11}=\gamma_{23}=1, \quad \gamma_{12}=\gamma_{13}=0 ; \quad \gamma_{2 n}=\rho_{n}, \quad n=1,2
$$

hence

$$
\begin{gathered}
\gamma_{11}=\left(a_{11} c_{11}+a_{12} c_{21}\right)\left(b_{11}\right)^{2}+\left(a_{11} c_{12}+a_{12} c_{22}\right) b_{11} b_{21} \\
+\left(a_{11} c_{13}+a_{12} c_{23}\right)\left(b_{21}\right)^{2}=1, \\
\gamma_{12}=2\left(a_{11} c_{11}+a_{12} c_{21}\right) b_{11} b_{12}+\left(a_{11} c_{12}+a_{12} c_{22}\right)\left(b_{11} b_{22}+b_{12} b_{21}\right) \\
+2\left(a_{11} c_{13}+a_{12} c_{23}\right) b_{21} b_{22}=0, \\
\gamma_{13}=\left(a_{11} c_{11}+a_{12} c_{21}\right)\left(b_{12}\right)^{2}+\left(a_{11} c_{12}+a_{12} c_{22}\right) b_{12} b_{22} \\
+\left(a_{11} c_{13}+a_{12} c_{23}\right)\left(b_{22}\right)^{2}=0,
\end{gathered}
$$




$$
\begin{gathered}
\gamma_{23}=\left(a_{21} c_{11}+a_{22} c_{21}\right)\left(b_{12}\right)^{2}+\left(a_{21} c_{12}+a_{22} c_{22}\right) b_{12} b_{22} \\
+\left(a_{21} c_{13}+a_{22} c_{23}\right)\left(b_{22}\right)^{2}=1, \\
\gamma_{21}=\left(a_{21} c_{11}+a_{22} c_{21}\right)\left(b_{11}\right)^{2}+\left(a_{21} c_{12}+a_{22} c_{22}\right) b_{11} b_{21} \\
+\left(a_{21} c_{13}+a_{22} c_{23}\right)\left(b_{21}\right)^{2}=\rho_{1}, \\
\gamma_{22}=2\left(a_{21} c_{11}+a_{22} c_{21}\right) b_{11} b_{12}+ \\
+\left(a_{21} c_{12}+a_{22} c_{22}\right)\left(b_{11} b_{22}+b_{12} b_{21}\right) \\
+2\left(a_{21} c_{13}+a_{22} c_{23}\right) b_{21} b_{22}=\rho_{2} ;
\end{gathered}
$$

namely, by setting,

$$
\alpha_{n \ell}=a_{n 1} c_{1 \ell}+a_{n 2} c_{2 \ell}, \quad n=1,2, \quad \ell=1,2,3,
$$

the following 6 equations:

$$
\begin{aligned}
& \alpha_{11}\left(b_{11}\right)^{2}+\alpha_{12} b_{11} b_{21}+\alpha_{13}\left(b_{21}\right)^{2}=1, \\
& 2 \alpha_{11} b_{11} b_{12}+\alpha_{12}\left(b_{11} b_{22}+b_{12} b_{21}\right)+2 \alpha_{13} b_{21} b_{22}=0, \\
& \alpha_{11}\left(b_{12}\right)^{2}+\alpha_{12} b_{12} b_{22}+\alpha_{13}\left(b_{22}\right)^{2}=0, \\
& \alpha_{21}\left(b_{12}\right)^{2}+\alpha_{22} b_{12} b_{22}+\alpha_{23}\left(b_{22}\right)^{2}=1, \\
& \alpha_{21}\left(b_{11}\right)^{2}+\alpha_{22} b_{11} b_{21}+\alpha_{23}\left(b_{21}\right)^{2}=\rho_{1}, \\
& 2 \alpha_{21} b_{11} b_{12}+\alpha_{22}\left(b_{11} b_{22}+b_{12} b_{21}\right)+2 \alpha_{23} b_{21} b_{22}=\rho_{2} .
\end{aligned}
$$

Remark 4-1. From the first 3 of these relations - summing the first and the third and summing or subtracting the second - we get the following 2 relations

$$
\alpha_{11}\left(b_{11} \pm b_{12}\right)^{2}+\alpha_{12}\left(b_{11} \pm b_{12}\right)\left(b_{21} \pm b_{22}\right)+\alpha_{13}\left(b_{21} \pm b_{22}\right)^{2}=1,
$$

and summing and subtracting these 2 relations we get the 2 relations

$$
\begin{aligned}
& \alpha_{11}\left[\left(b_{11}\right)^{2}+\left(b_{12}\right)^{2}\right]+\alpha_{12}\left(b_{11} b_{21}+b_{12} b_{22}\right)+\alpha_{13}\left[\left(b_{21}\right)^{2}+\left(b_{22}\right)^{2}\right]=1, \\
& 2 \alpha_{11} b_{11} b_{12}+\alpha_{12}\left(b_{11} b_{22}+b_{12} b_{21}\right)+2 \alpha_{13} b_{21} b_{22}=0 .
\end{aligned}
$$

But we shall not use these formulas below. 
Solving the first 3 of the 6 eqs. (22) we get the following formulas for the 3 quantities $\alpha_{1 \ell}(\ell=1,2,3)$ :

$$
\alpha_{11}=\left(b_{22}\right)^{2} / B^{2}, \alpha_{12}=-2 b_{12} b_{22} / B^{2}, \alpha_{13}=\left(b_{12}\right)^{2} / B^{2},
$$

and likewise solving the last 3 of the 6 eqs. (22) we get the following formulas for the 3 quantities $\alpha_{2 \ell}(\ell=1,2,3)$ :

$$
\begin{aligned}
& \alpha_{21}=\left[\left(b_{21}\right)^{2}+\left(b_{22}\right)^{2} \rho_{1}-b_{21} b_{22} \rho_{2}\right] / B^{2}, \\
& \alpha_{22}=-\left[2 b_{11} b_{21}+2 b_{12} b_{22} \rho_{1}-\left(b_{11} b_{22}+b_{12} b_{21}\right) \rho_{2}\right] / B^{2}, \\
& \alpha_{23}=\left[\left(b_{11}\right)^{2}+\left(b_{12}\right)^{2} \rho_{1}-b_{11} b_{12} \rho_{2}\right] / B^{2} ;
\end{aligned}
$$

of course, above and below, $B$ is defined in terms of the 4 parameters $b_{n m}$ by eq. (5d).

Next, using the definitions (21) and the relations (5a), we get the following 6 algebraic equations, which only involve the 6 parameters $\rho_{1}, \rho_{2}$ and $b_{n m}$ as well as the 6 parameters $c_{n \ell}$ :

$$
\begin{aligned}
& \left(b_{22} c_{11}-b_{12} c_{21}\right) B=\left(b_{22}\right)^{2}, \\
& \left(b_{22} c_{12}-b_{12} c_{22}\right) B=-2 b_{12} b_{22}, \\
& \left(b_{22} c_{13}-b_{12} c_{23}\right) B=\left(b_{12}\right)^{2}, \\
& \left(-b_{21} c_{11}+b_{11} c_{21}\right) B=\left(b_{21}\right)^{2}+\left(b_{22}\right)^{2} \rho_{1}-b_{21} b_{22} \rho_{2}, \\
& \left(b_{21} c_{12}-b_{11} c_{22}\right) B=2 b_{11} b_{21}+2 b_{12} b_{22} \rho_{1}-\left(b_{11} b_{22}+b_{12} b_{21}\right) \rho_{2}, \\
& \left(-b_{21} c_{13}+b_{11} c_{23}\right) B=\left(b_{11}\right)^{2}+\left(b_{12}\right)^{2} \rho_{1}-b_{11} b_{12} \rho_{2} .
\end{aligned}
$$

In all these formulas $B$ is of course again defined in terms of the 4 parameters $b_{n m}$ by the formula $(\underline{5 d})$.

Solving for $\rho_{1}$ and $\rho_{2}$ the 2 linear eqs. (27d) and (27e) we get

$$
\begin{aligned}
& \rho_{1}=\left\{\left(b_{21}\right)^{2}\left(1-b_{12} c_{11}-b_{22} c_{12}\right)+\left(b_{11}\right)^{2} b_{22} c_{21}\right. \\
&\left.+b_{11} b_{21}\left[b_{12} c_{21}-b_{22}\left(c_{11}-c_{22}\right)\right]\right\} /\left(b_{22}\right)^{2}, \\
& \rho_{2}=\left(2 b_{21}-2 b_{12} b_{21} c_{11}-b_{21} b_{22} c_{12}+2 b_{11} b_{12} c_{21}+b_{11} b_{22} c_{22}\right) / b_{22} ;
\end{aligned}
$$

likewise, solving the 2 linear eqs. (27e $)$ and $(27 \mathrm{f})$, we get

$$
\begin{aligned}
\rho_{1}= & \left\{b_{12}\left(b_{21}\right)^{2} c_{13}+b_{11} b_{21}\left[b_{22} c_{13}+b_{12}\left(c_{12}-c_{23}\right)\right]\right. \\
& \left.+\left(b_{11}\right)^{2}\left(1-b_{12} c_{22}-b_{22} c_{23}\right)\right\} /\left(b_{12}\right)^{2}
\end{aligned}
$$




$$
\rho_{2}=\left[b_{11}\left(2-b_{12} c_{22}-2 b_{22} c_{23}\right)+b_{12} b_{21} c_{12}+2 b_{21} b_{22} c_{13}\right] / b_{12} ;
$$

and likewise, solving the 2 linear eqs. (27f) and (27d), we get

$$
\begin{aligned}
& \rho_{1}=\left[\left(b_{21}\right)^{2} b_{22} c_{13}+\left(b_{11}\right)^{2} b_{12} c_{21}+b_{11} b_{21}\left(1-b_{12} c_{11}-b_{22} c_{23}\right)\right] /\left(b_{12} b_{22}\right), \\
& \rho_{2}=b_{21} / b_{22}+\left[b_{12}\left(-b_{21} c_{11}+b_{11} c_{21}\right)\right] / b_{22}+\left(b_{11}+b_{21} b_{22} c_{13}-b_{11} b_{22} c_{23}\right) / b_{12} .
\end{aligned}
$$

Any one of these 3 pairs of formulas provides an explicit expression of the 2 parameters $\rho_{1}$ and $\rho_{2}$ in terms of the 4 parameters $b_{n m}$ and the 6 parameters $c_{n \ell}$. Hence hereafter we may only focus on the problem to express the 4 parameters $b_{n m}$ in terms of the 6 parameters $c_{n \ell}$.

Indeed, by identifying 2 different expressions of the parameter $\rho_{1}$ or $\rho_{2}$ as given just above, we obtain additional formulas involving only the 4 parameters $b_{n m}$ and the 6 parameters $c_{n \ell}$. In particular by identifying the 2 expressions $(28 \mathrm{~b})$ and $(29 \mathrm{~b})$ we get the following formula:

$$
\begin{aligned}
& b_{12}\left(2 b_{21}-2 b_{12} b_{21} c_{11}-b_{21} b_{22} c_{12}+2 b_{11} b_{12} c_{21}+b_{11} b_{22} c_{22}\right) \\
= & b_{22}\left[b_{11}\left(2-b_{12} c_{22}-2 b_{22} c_{23}\right)+b_{12} b_{21} c_{12}+2 b_{21} b_{22} c_{13}\right]
\end{aligned}
$$

and likewise by identifying the 2 expressions (29a) and (30a) we get the following formula:

$$
\begin{aligned}
& b_{22}\left\{b_{11} b_{21}\left[b_{22} c_{13}+b_{12}\left(c_{12}-c_{23}\right)\right]+\left(b_{11}\right)^{2}\left(1-b_{12} c_{22}-b_{22} c_{23}\right)\right\} \\
= & b_{12}\left[\left(b_{11}\right)^{2} b_{12} c_{21}+b_{11} b_{21}\left(1-b_{12} c_{11}-b_{22} c_{23}\right)\right] .
\end{aligned}
$$

Our final task is to extract as much information as possible on the dependence of the 4 parameters $b_{n m}$ on the 6 parameters $c_{n \ell}$, from these 2 equations (31) and from the 3 eqs. (27a), (27b), (27c), or rather from 2 of their 3 ratios, which clearly read as follows:

$$
\begin{aligned}
& -2 b_{12}\left(b_{22} c_{11}-b_{12} c_{21}\right)=b_{22}\left(b_{22} c_{12}-b_{12} c_{22}\right), \\
& b_{12}\left(b_{22} c_{12}-b_{12} c_{22}\right)=-2 b_{22}\left(b_{22} c_{13}-b_{12} c_{23}\right), \\
& \left(b_{12}\right)^{2}\left(b_{22} c_{11}-b_{12} c_{21}\right)=\left(b_{22}\right)^{2}\left(b_{22} c_{13}-b_{12} c_{23}\right) ;
\end{aligned}
$$

each one of these 3 formulas (32) is of course implied by the other 2 .

Let us now introduce the auxiliary variable

$$
\beta=b_{12} / b_{22}
$$

Then, by dividing the 2 eqs.(32a $)$ and $(32 \mathrm{~b})$ by $\left(b_{22}\right)^{2}$ we get the following 2 quadratic equations for this quantity:

$$
\begin{aligned}
& 2 c_{21} \beta^{2}+\left(c_{22}-2 c_{11}\right) \beta-c_{12}=0, \\
& c_{22} \beta^{2}-\left(c_{12}-2 c_{23}\right) \beta-2 c_{13}=0 .
\end{aligned}
$$


Subtracting the second of these 2 eqs. multiplied by $2 c_{21}$ from the first itself multiplied by $c_{22}$ we get a first-degree equation for $\beta$, the solution of which reads

$$
\beta=\left(c_{12} c_{22}-4 c_{13} c_{21}\right) /\left[\left(c_{22}-2 c_{11}\right) c_{22}+2 c_{21}\left(c_{12}-2 c_{23}\right)\right] ;
$$

and inserting this determination of $\beta$ in the 2 eqs. (34) we finally get the following 2 explicit constraints on the 6 coefficients $c_{n \ell}$ :

$$
\begin{array}{r}
2 c_{21}\left(c_{12} c_{22}-4 c_{13} c_{21}\right)^{2} \\
+\left(c_{22}-2 c_{11}\right)\left(c_{12} c_{22}-4 c_{13} c_{21}\right)\left[\left(c_{22}-2 c_{11}\right) c_{22}+2 c_{21}\left(c_{12}-2 c_{23}\right)\right] \\
-c_{12}\left[\left(c_{22}-2 c_{11}\right) c_{22}+2 c_{21}\left(c_{12}-2 c_{23}\right)\right]^{2}=0 \\
c_{22}\left(c_{12} c_{22}-4 c_{13} c_{21}\right)^{2} \\
-\left(c_{12}-2 c_{23}\right)\left(c_{12} c_{22}-4 c_{13} c_{21}\right)\left[\left(c_{22}-2 c_{11}\right) c_{22}+2 c_{21}\left(c_{12}-2 c_{23}\right)\right] \\
-2 c_{13}\left[\left(c_{22}-2 c_{11}\right) c_{22}+2 c_{21}\left(c_{12}-2 c_{23}\right)\right]^{2}=0 .
\end{array}
$$

These 2 constraints on the 6 coefficients $c_{n \ell}$ must be satisfied in order that the initialvalues problem of the system (11) be explicitly solvable as detailed by Proposition 2-2. Note that each of these constraints is a quintic algebraic equation for the 6 coefficients $c_{n \ell}$; but eq. (36a) is only quadratic for $c_{11}, c_{13}, c_{23}$ and cubic for $c_{12}, c_{21}, c_{22}$; while eq. (36b) is only quadratic for $c_{11}, c_{13}, c_{21}, c_{23}$, cubic for $c_{12}$ and quartic for $c_{22}$.

Remark 4.2. The last sentence above suggests the most convenient approaches to be employed in order to evaluate the implications of the 2 constraints (36) in the special cases - generally relevant in applicative contexts - when the 6 coefficients $c_{n \ell}$ are all real numbers.

Let us now complete the task of this Section, to express the 4 parameters $b_{n m}$-hence as well the 4 parameters $a_{n m}$ : see (5a) with (5d) -in terms of the 6 coefficients $c_{n \ell}$. Since the definition (33) of $\beta$ clearly implies

$$
b_{12}=\beta b_{22},
$$

inserting this relation in the 3 eqs. (27a), (31a) and (31b), we get the following 3 algebraic equations:

$$
\begin{aligned}
& \left(c_{11}-\beta c_{21}\right)\left(b_{11}-\beta b_{21}\right)=1, \\
& {\left[1-b_{22}\left(\beta^{2} c_{21}+\beta c_{22}+c_{23}\right)\right] b_{11}} \\
& +\left[-\beta+b_{22}\left(\beta^{2} c_{11}+\beta c_{12}+c_{13}\right)\right] b_{21}=0, \\
& b_{21} b_{22}\left[c_{13}+\beta\left(c_{12}-c_{23}\right)\right]+\left(b_{11}\right)^{2}\left[1-b_{22}\left(\beta c_{22}+c_{23}\right)\right] \\
& \quad-\beta\left\{\beta b_{11} b_{22} c_{21}+b_{21}\left[1-b_{22}\left(\beta c_{11}+c_{23}\right)\right]\right\}=0 .
\end{aligned}
$$

The first 2 of these 3 eqs. (38) are a linear system for the 2 quantities $b_{11}$ and $b_{22}$, which can be immediately solved yielding

$$
b_{11}=B_{110}+\beta b_{21},
$$




$$
b_{22}=1 /\left(B_{220}+B_{221} b_{21}\right)
$$

with

$$
\begin{aligned}
& B_{110}=1 /\left(c_{11}-\beta c_{21}\right), \\
& B_{220}=c_{23}+\beta c_{22}+\beta^{2} c_{21}, \\
& B_{221}=-\left(c_{11}-\beta c_{21}\right)\left[c_{13}+\beta\left(c_{12}-c_{23}\right)+\beta^{2}\left(c_{11}-c_{22}\right)-c_{21} \beta^{3}\right] .
\end{aligned}
$$

Note that, since $\beta$ is explicitly expressed in terms of the 6 coefficients $c_{n \ell}$ (see (35)), the 2 formulas (39) - together with (37) - provide explicit expressions of the 3 parameters $b_{11}$, $b_{22}, b_{12}$ in terms of the 6 coefficients $c_{n \ell}$ and the parameter $b_{21}$. Thus to complete our task we must express also this parameter $b_{21}$ in terms of the 6 coefficients $c_{n \ell}$. This task can be fulfilled by solving the algebraic equation (38c), which-after the replacement of the 3 parameters $b_{11}, b_{22}, b_{21}$ via their expressions (37) and (39) in terms of $b_{21}$ and the 6 parameters $c_{n \ell}$-only features the still unknown parameter $b_{21}$ (of course in addition to the 6 parameters $c_{n \ell}$ ). And it can be shown-via elementary if tedious calculations, which can be checked by Mathematica - that the eq. (38c) takes then the following form:

$$
C_{0}+C_{1} b_{21}+C_{2}\left(b_{21}\right)^{2}+C_{3}\left(b_{21}\right)^{3}=0
$$

with

$$
\begin{aligned}
& C_{0}=\beta^{2} c_{21}\left(1-c_{11}+\beta c_{21}\right), \\
& C_{1}=\left(c_{11}-\beta c_{21}\right)\left\{\beta^{3} c_{21}-\left(1-c_{11}+\beta c_{21}\right) .\right. \\
& \left.\cdot\left[c_{13}+\beta\left(c_{12}-c_{23}\right)+\beta^{2}\left(c_{11}-c_{22}\right)-2 \beta^{3} c_{21}\right]\right\}, \\
& C_{2}=-\beta\left(c_{11}-\beta c_{21}\right)^{2}\left\{c_{13}+\beta\left(c_{12}-c_{23}\right)+\beta^{2}\left(c_{11}-c_{22}\right)-2 \beta^{3} c_{21}\right. \\
& \left.+\left(1-c_{11}+\beta c_{21}\right)\left[c_{13}-\beta\left(c_{23}-c_{12}\right)+\beta^{2}\left(c_{11}-c_{22}\right)-\beta^{3} c_{21}\right]\right\}, \\
& C_{3}=-\beta^{2}\left(c_{11}-\beta c_{21}\right)^{3}\left[c_{13}+\beta\left(c_{12}-c_{23}\right)+\beta^{2}\left(c_{11}-c_{22}\right)-\beta^{3} c_{21}\right] .
\end{aligned}
$$

Since these 4 coefficients $C_{k}(k=0,1,2,3)$ are all explicitly expressed-via these formulas: see (35) and (40) - in terms of the 6 coefficients $c_{n \ell}$, it seems that to complete our task all that still needs to be done is to solve the cubic equation (40a), which can of course be explicitly solved via the Cardano formulas.

But the situation is a bit more tricky, and in fact more simple.

The point is that, as we know, the 6 parameters $c_{n \ell}$ cannot be assigned freely; the success of the entire treatment requires that they satisfy the 2 constraints (36); and, as it happens, this requirement seems to imply that the coefficient $C_{3}$ vanishes, $C_{3}=0$. We have been unable to prove this result explicitly: note that the expression of $C_{3}$ in terms 
of the 6 coefficients $c_{n \ell}$ is quite complicated, also due to the complicated dependence of $\beta$ on the coefficients $c_{n \ell}$ (see (35)); and the 2 constraints (36) are as well fairly complicated. But quite convincing evidence of this fact is provided by the numerical examples reported below, see Subsection 4.1.

Hence, the third-degree equation (40a) can be replaced by the second-degree equation

$$
C_{0}+C_{1} b_{21}+C_{2}\left(b_{21}\right)^{2}=0
$$

the 2 solutions of which read of course as follows:

$$
b_{21}=b_{21 \pm} \equiv\left(-C_{1} \pm \sqrt{\left(C_{1}\right)^{2}-4 C_{0} C_{2}}\right) / 2 .
$$

This finding seems to complete our task to determine - in terms of 6 coeffcients $c_{n \ell}$, arbitrarily assigned except for the requirement to satisfy the 2 constraints (36) - the 6 parameters $\rho_{n}$ and $b_{n m}(n, m=1,2)$ : see (35), (29), (37), (39) and (41b). Hence to provide the explicit solution of the initial-values problem of the system (11), as detailed by Proposition 2-2 in terms of the 6 parameters $\rho_{n}$ and $b_{n m}$.

But a doubt should still linger in the mind of the alert reader: the solution of the initial-values problem of the system (11) should be unique; but we just found 2 different values for the parameter $b_{21}$ (see (41b) ), hence as well for the other 3 parameters $b_{n m}$ (see the eqs. (39) and (37)), and as well for the parameters $\rho_{n}$ (see the eqs. (29)). This means that, if our treatment is correct, these different values must end up yielding the same solution for the variables $x_{n}(t)$. This "miracle" is indeed validated by a check of many specific examples, as reported in the following Subsection 4.1; with the added observation that - as implied by the observation that the eqs. (39e) and (40e) clearly imply $B_{221}=C_{3}\left[\beta\left(c_{11}-\beta c_{21}\right)\right]^{-2}$ — we may conclude that the vanishing of the parameter $C_{3}$ also implies the vanishing of the parameter $B_{221}: B_{221}=0$; implying (via (39d)) the replacement of the expression (39b) of $b_{22}$ by the simple expression

$$
b_{22}=1 /\left(c_{23}+\beta c_{22}+\beta^{2} c_{21}\right),
$$

and as a consequence also the replacement of (37) with

$$
b_{12}=\beta /\left(c_{23}+\beta c_{22}+\beta^{2} c_{21}\right) \text {. }
$$

These simpler formulas expressing the 2 parameters $b_{22}$ and $b_{12}$ directly via the parameters $c_{n \ell}$ (recall (35) ) imply that the values of these 2 parameters are not affected by the 2 -valued indeterminacy affecting the other 2 parameters $b_{11}$ and $b_{21}$ (see (39a) and (41b)) as well as the values of the 2 parameters $\rho_{n}$ (see (29) $)$.

\subsection{Specific solvable examples}

Let us introduce this Subsection by emphasizing that - due to the explicit character of the formulas (4) expressing the 6 coefficients $c_{n \ell}$ in terms of the 6 parameters $\rho_{n}$ and $b_{n m}$ (or, equivalently, $a_{n m}$ : see (5) - it is quite easy to manufacture examples of the system (10) which are explicitly solvable via our treatment: all one has to do is to input an arbitrary assignment of these 6 parameters $\rho_{n}$ and $b_{n m}$ in these formulas (4). 
In this Subsection we report only 3 examples of the system (1) which are explicitly solvable via the technique described in the present paper. But we also tested several other such examples, which are not reported here; they all confirmed the assertion (that $C_{3}=0$ ) mentioned in the last part of Section 4 . Of course it shall be likewise easy for the interested reader to identify in this manner other systems (1) explicitly solvable via the technique introduced in this paper (see Propositions 2- 2 and 2-1).

By inserting the values of the parameters $c_{n \ell}$-obtained by the simple procedure described in the first paragraph of this Subsection - in the relevant formulas written above (see Section 4), we verified that they of course do satisfy the 2 constraints (36); that they always do yield a vanishing value for the parameter $C_{3}$ (and also for the parameter $B_{221}$ ); we obtained specific values for each of the 2 parameters $b_{22}$ and $b_{12}$ (of course, the same as those originally employed to determine the set of coefficients $c_{n \ell}$ ); while we obtained instead 2 alternative determinations for the couple of parameters $b_{11}$ and $b_{21}$ and also for the couple of parameters $\rho_{1}$ and $\rho_{2}$. And moreover - remarkably: although this "miracle" was expected - we verified that these 2 different determinations yield - via the relevant formulas of Proposition 2-2 and 2-1 (see eqs. (6b), (3a), (42), (41b), (39a), (29)) - the same, unique, solution of the initial-values problem of the system (11).

The first example is identified by the following assignments of the 6 coefficients $c_{n \ell}$ :

$$
c_{11}=7 / 3, \quad c_{12}=2, \quad c_{13}=3, \quad c_{21}=-1, \quad c_{22}=-2, \quad c_{23}=-3 .
$$

The corresponding values of the parameters $\beta, b_{12}$ and $b_{22}$ are

$$
\beta=-3, \quad b_{12}=1 / 2, \quad b_{22}=-1 / 6
$$

while for the values of the parameters $b_{11}, b_{21}, \rho_{1}, \rho_{2}$ and $\Delta$ (see (3e) ) we get

$$
b_{11}=0, \quad b_{21}=-1 / 2, \quad \rho_{1}=3 / 2, \quad \rho_{2}=0, \quad \Delta=\mathbf{i} \sqrt{5},
$$

or

$$
b_{11}=1, \quad b_{21}=-5 / 6, \quad \rho_{1}=7 / 2, \quad \rho_{2}=4, \quad \Delta=\mathbf{i} \sqrt{5}
$$

note the equality of the 2 determinations of the parameter $\Delta$, which are of course essential for the final outcome, namely the following unique explicit solution of the initial-values problem of the system (1) with (43a):

$$
\begin{array}{r}
x_{1}(t)=\mathbf{i}\left\{3 [ x _ { 1 } ( 0 ) + 3 x _ { 2 } ( 0 ) ] \left[(2-\mathbf{i} \sqrt{5}) x_{1}(0)+3 x_{2}(0)\right.\right. \\
\left.-\left[(2+\mathbf{i} \sqrt{5}) x_{1}(0)+3 x_{2}(0)\right]\left[1+(2 / 3) t\left(x_{1}(0)+3 x_{2}(0)\right]^{\mathbf{i} \sqrt{5}}\right]\right\} / D_{1}(t) \\
x_{2}(t)=\left\{3 [ x _ { 1 } ( 0 ) + 3 x _ { 2 } ( 0 ) ] \left[-3 x_{1}(0)-2 x_{2}(0)-\mathbf{i} \sqrt{5} x_{2}(0)\right.\right. \\
\left.\left.+\left[3 x_{1}(0)+(2-\mathbf{i} \sqrt{5}) x_{2}(0)\right]\left\{1+(2 / 3) t\left[x_{1}(0)+3 x_{2}(0)\right]\right\}^{\mathbf{i} \sqrt{5}}\right]\right\} / D_{1}(t)
\end{array}
$$




$$
\begin{aligned}
& D_{1}(t)=\left\{3+2 t\left[x_{1}(0)+3 x_{2}(0)\right]\right\}\left[(-7-\mathbf{i} \sqrt{5}) x_{1}(0)+(-3-3 \mathbf{i} \sqrt{5}) x_{2}(0)\right. \\
& \left.+\left[(7-\mathbf{i} \sqrt{5}) x_{1}(0)+3(1-\mathbf{i} \sqrt{5}) x_{2}(0)\right]\left\{1+(2 / 3) t\left[x_{1}(0)+3 x_{2}(0)\right]\right\}^{\mathbf{i} \sqrt{5}}\right] .
\end{aligned}
$$

The second example is identified by the following assignments of the 6 coefficients $c_{n \ell}$ :

$$
c_{11}=c_{12}=c_{13}=1, \quad c_{21}=1 / 8, \quad c_{22}=2, \quad c_{23}=-1 .
$$

The corresponding data read then as follows:

$$
\beta=2, \quad b_{12}=4 / 7, \quad b_{22}=2 / 7,
$$

and

$$
b_{11}=0, \quad b_{21}=-2 / 3, \quad \rho_{1}=7 / 9, \quad \rho_{2}=-4 / 3, \quad \Delta=\sqrt{7 / 3},
$$

or

$$
b_{11}=1, \quad b_{21}=-1 / 6, \quad \rho_{1}=-35 / 144, \quad \rho_{2}=13 / 6, \quad \Delta=\sqrt{7 / 3} ;
$$

yielding the following unique explicit solution of the initial-values problem of the system (11) with (44a):

$$
\begin{gathered}
x_{1}(t)=\left\{4\left[x_{1}(0)-2 x_{2}(0)\right]\left[(5-\sqrt{21}) x_{1}(0)+4 x_{2}(0)\right]\right. \\
\left.-\left[(5+\sqrt{21}) x_{1}(0)+4 x_{2}(0)\right]\left\{1-(3 / 4)\left[x_{1}(0)-2 x_{2}(0)\right] t\right\}^{\sqrt{7 / 3}}\right\} / D_{2}(t) \\
\left.+\left[x_{1}(0)+(5-\sqrt{21}) x_{2}(0)\right]\left\{1-(3 / 4)\left[x_{1}(0)-2 x_{2}(0)\right] t\right\}^{\sqrt{7 / 3}}\right\} / D_{2}(t) \\
D_{2}(t)=\left\{-4+3\left[x_{1}(0)-2 x_{2}(0)\right] t\right\}\left\{(-7+\sqrt{21}) x_{1}(0)-2(7+\sqrt{21}) x_{2}(0)\right. \\
\left.+\left[(7+\sqrt{21}) x_{1}(0)+2(7-\sqrt{21}) x_{2}(0)\right]\left\{1-(3 / 4)\left[x_{1}(0)-2 x_{2}(0)\right] t\right\}^{\sqrt{7 / 3}}\right\}
\end{gathered}
$$

The third example is identified by the following assignments of the 6 coefficients $c_{n \ell}$ :

$$
\begin{aligned}
& c_{11}=-19 / 169, \quad c_{12}=-265 / 507, \quad c_{13}=110 / 1521, \\
& c_{21}=-27 / 169, \quad c_{22}=-1 / 169, \quad c_{23}=-36 / 169 .
\end{aligned}
$$

The corresponding data read then as follows:

$$
\beta=5 / 3, \quad b_{12}=-5 / 2, \quad b_{22}=-3 / 2,
$$


and

$$
b_{11}=0, \quad b_{21}=-39 / 10, \quad \rho_{1}=-11 / 25, \quad \rho_{2}=17 / 10, \quad \Delta=3 / 2,
$$

or

$$
b_{11}=1, \quad b_{21}=-33 / 10, \quad \rho_{1}=-14 / 25, \quad \rho_{2}=9 / 10, \quad \Delta=3 / 2 ;
$$

yielding the following unique explicit solution of the initial-values problem of the system (11) with (44a):

$$
\begin{gathered}
x_{1}(t)=\left\{[ 3 x _ { 1 } ( 0 ) - 5 x _ { 2 } ( 0 ) ] \left\{3861 x_{1}(0)-858 x_{2}(0)\right.\right. \\
\left.+78\left[9 x_{1}(0)+11 x_{2}(0)\right]\left\{1-(2 / 39)\left[3 x_{1}(0)-5 x_{2}(0)\right] t\right\}^{3 / 2}\right\} / D_{3}(t), \\
x_{2}(t)=-\left\{9 [ 3 x _ { 1 } ( 0 ) - 5 x _ { 2 } ( 0 ) ] \left\{351 x_{1}(0)-78 x_{2}(0)\right.\right. \\
\left.-39\left[9 x_{1}(0)+11 x_{2}(0)\right]\left\{1-(2 / 39)\left[3 x_{1}(0)-5 x_{2}(0)\right] t\right\}^{3 / 2}\right\} / D_{3}(t), \\
D_{3}(t)=\left\{39-2\left[3 x_{1}(0)-5 x_{2}(0)\right] t\right\}\left\{702 x_{1}(0)-156 x_{2}(0)\right. \\
\left.-39\left[9 x_{1}(0)+11 x_{2}(0)\right]\left\{1-(2 / 39)\left[3 x_{1}(0)-5 x_{2}(0)\right] t\right\}^{3 / 2}\right\} .
\end{gathered}
$$

\section{$5 \quad$ Invariance property and simplifications}

In this short section we report for completeness a rather obvious invariance property and some possible trivial simplifications of the system (1). They amount to the elementary observation that the 2 dependent variables

$$
\hat{x}_{n}(\tau) \equiv\left(\mu_{n} / \lambda\right) x_{n}(t), \quad \hat{t} \equiv \lambda t
$$

with $\lambda$ and $\mu_{n}$ a priori arbitrary nonvanishing parameters, satisfy-mutatis mutandisessentially the same system (11) as the 2 dependent variables $x_{n}(t)$ :

$$
\hat{x}_{n}^{\prime}(\hat{t}) \equiv d \hat{x}_{n}(\hat{t}) / d \hat{t}=\hat{c}_{n 1}\left[\hat{x}_{1}(\hat{t})\right]^{2}+\hat{c}_{n 2} \hat{x}_{1}(\hat{t}) \hat{x}_{2}(\hat{t})+\hat{c}_{n 3}\left[\hat{x}_{2}(\hat{t})\right]^{2}, \quad n=1,2,
$$

with

$$
\hat{c}_{n 1}=\mu_{n}\left(\mu_{1}\right)^{-2} c_{n 1}, \quad \hat{c}_{n 2}=\mu_{n}\left(\mu_{1} \mu_{2}\right)^{-1} c_{n 2}, \quad \hat{c}_{n 3}=\mu_{n}\left(\mu_{2}\right)^{-2} c_{n 3}, \quad n=1,2 .
$$

For $\mu_{1}=\mu_{2}=1$ this property identifies the invariance of the system (11) under a simultaneous rescaling of the independent and dependent variables: see (46).

Remark 5-1. Both constraints (36) are invariant under the transformation (46c).

The simplifications correspond to the possibility to replace - by an appropriate rescaling of dependent variables -1 of the 3 parameters $c_{1 \ell}$ and 1 of the 3 parameters $c_{2 \ell}$ by an 
arbitrary number (of course, nonvanishing; for instance, just unity); thereby reducing the number of a priori arbitrary coefficients $c_{n \ell}$ from 6 to 4 . For instance the assignment

$$
\mu_{1}=c_{11}, \quad \mu_{2}=c_{23},
$$

implies

$$
\begin{aligned}
& \hat{c}_{11}=1, \quad \hat{c}_{12}=c_{12} / c_{23}, \quad \hat{c}_{13}=c_{11} c_{13}\left(c_{23}\right)^{-2}, \\
& \hat{c}_{21}=c_{21} c_{23}\left(c_{11}\right)^{-2}, \quad \hat{c}_{22}=c_{22} / c_{11}, \quad \hat{c}_{23}=1 .
\end{aligned}
$$

Remark 5-2. The simplification (47) applied to the 3 examples characterized by the assignments (43), (44) respectively (45) yields 3 models (see (46)) characterized by the following assignments of the coefficients $c_{n \ell}$ :

$$
\hat{c}_{12}=-2 / 3, \quad \hat{c}_{13}=7 / 9, \quad \hat{c}_{21}=27 / 49, \quad \hat{c}_{22}=-6 / 7,
$$

respectively

$$
\hat{c}_{12}=-1, \quad \hat{c}_{13}=1, \quad \hat{c}_{21}=-1 / 8, \quad \hat{c}_{22}=2,
$$

respectively

$$
\hat{c}_{12}=\frac{265}{108}, \quad \hat{c}_{13}=-\frac{1045}{5832}, \quad \hat{c}_{21}=\frac{972}{361}, \quad \hat{c}_{22}=\frac{1}{19} ;
$$

of course in all 3 cases with $\hat{c}_{11}=\hat{c}_{23}=1$.

\section{Extensions and isochronous models}

In this Section we tersely outline some simple extensions of the system (1) to the case with non-homogeneous quadratic right-hand sides, as well as some related systems obtained by a well-known change of variables - see for instance [1] - which allows the identification of analogous systems featuring the remarkable property to be isochronous.

An elementary way to extend the autonomous system (11) featuring ODEs with homogeneous quadratic right-hand sides to an, also autonomous, system with non-homogeneous quadratic right-hand sides is via the following - easily invertible - change of independent variables:

$$
z_{n}(t)=\exp (\eta t) x_{n}(\tilde{t})+\bar{z}_{n}, \quad \tilde{t}=[\exp (\eta t)-1] / \eta, \quad n=1,2,
$$

where the 3 parameters $\bar{z}_{1}, \bar{z}_{2}$ and $\eta$ are a priori arbitrary. Thereby the system (11) gets transformed into the following system:

$$
\begin{aligned}
& \dot{z}_{n}(t)=c_{n 1}\left[z_{1}(t)\right]^{2}+c_{n 2} z_{1}(t) z_{2}(t)+c_{n 3}\left[z_{2}(t)\right]^{2} \\
& +\eta z_{n}(t)+d_{n 1} z_{1}(t)+d_{n 2} z_{2}(t)+d_{n 3}, \quad n=1,2,
\end{aligned}
$$

with the 6 "new" parameters $d_{n \ell}$ expressed in terms of the 6 "old" parameters $c_{n \ell}$ and of the 3 "new" parameters $\bar{z}_{1}, \bar{z}_{2}$ and $\eta$ as follows:

$$
\begin{aligned}
& d_{n 1}=-2 c_{n 1} \bar{z}_{1}-c_{n 2} \bar{z}_{2}, \quad d_{n 2}=-2 c_{n 1} \bar{z}_{2}-c_{n 2} \bar{z}_{1}, \\
& d_{n 3}=-\eta \bar{z}_{n}+c_{n 1}\left(\bar{z}_{1}\right)^{2}+c_{n 2} \bar{z}_{1} \bar{z}_{2}+c_{n 3}\left(\bar{z}_{2}\right)^{2}, \quad n=1,2 .
\end{aligned}
$$


Of course the solvability properties of the original system (11) carry over to the system (52).

Moreover - if the solvability of the system (52a), via (51) and Propositions 2-2 and 2-1 - features a parameter $\Delta$ (see (3e) ) which is a real rational number $\left(\Delta=k_{1} / k_{2}\right.$ with $k_{1}$ an arbitrary integer and $k_{2}$ an arbitrary positive integer), then clearly the system (52a), with

$$
\eta=\mathbf{i} \omega
$$

- where $\mathbf{i}$ is the imaginary unit, $\mathbf{i}^{2}=-1$, and $\omega$ is an arbitrary nonvanishing real numberfeatures the remarkable property to be isochronous: namely all its solutions $z_{n}(t)$ are periodic with the same period $T=2 \pi k_{2} /|\omega|$,

$$
z_{n}(t+T)=z_{n}(t), \quad n=1,2 .
$$

Readers wondering about the validity of this - rather obvious: see (51), (6), (3c) and (53) - conclusion are advised to have a look, for instance, at the book [1].

Remark 6-1. Of course the presence of the imaginary parameter $\eta=\mathbf{i} \omega$ in the righthand side of the system (52a) with (53) implies that its solutions are necessarily complex, $z_{n}(t) \equiv \operatorname{Re}\left[z_{n}(t)\right]+\mathbf{i} \operatorname{Im}\left[z_{n}(t)\right]$; entailing a corresponding doubling, from 2 to 4 , of the number of nonlinearly-coupled ODEs for the real version of this system, satisfied by the 4 real dependent variables $\operatorname{Re}\left[z_{n}(t)\right]$ and $\operatorname{Im}\left[z_{n}(t)\right], n=1,2$; and clearly in this case it would be natural to also consider the 6 parameters $c_{n \ell}$ (as well of course as the 6 parameters $\rho_{n}$ and $b_{n m}$ related to them) and the 2 parameters $\bar{z}_{n}$ to be themselves complex numbers.

Remark 6-2. The interested reader might wish to compute the relevant formulas for the isochronous case associated to the third example reported in Subsection 4.1.

\section{Comparison with previous findings and outlook}

The system (11) treated in this paper is identical to the system treated in the recent paper [2]; it is therefore appropriate to compare the approach and the findings reported in that paper with those reported in the present paper.

The methodologies used in [2] and in the present paper have much in common, but there is a significant difference. In the present paper we started from the simpler, explicitly solvable model (2) and we then investigated in which cases the general system (11) with 6 a priori arbitrary coefficients $c_{n \ell}$ can be reduced-via a time-independent linear transformation of the 2 dependent variables, see (6) - to the simpler, explicitly solvable system (2). We found that this is indeed possible, but only if the 6 a priori arbitrary coefficients $c_{n \ell}$ satisfy the 2 constraints (36). This allowed us to conclude that the special subclass of the systems (11) identified by these 2 constraints is explicitly solvable in terms of elementary functions, and to display the solution of their initial-values problem.

The methodology employed in [2] took as point of departure the general system (11) with 6 arbitrary coefficients $c_{n \ell}$, but then immediately proceeded to reduce it to a canonical form - featuring at most only 2 coefficients - via a time-independent linear transformation

of the 2 dependent variables (such as (6) ); it then focussed on the discussion of the solvability (by quadratures) of those reduced systems, and moreover on the identification of 
a specific subclass of such systems the solutions of which are algebraic, i. e. identified as roots of explicitly time-dependent polynomials. The procedure of reduction to canonical form is a bit complicated, but it has been shown by François Leyvraz that the first example treated in Subsection III.B of [2] (see eqs. (38-41 there) is essentially equivalent-up to notational changes - to the model treated in the present paper. We also take this opportunity to mention a trivial misprint in eq. (10b) of [2], which identifies the Newtonian equation $\ddot{\zeta}=\zeta^{k}$ as algebraically solvable if $k=-(2 n+1) /(2 n-1)$ or $k=-(n+1) / n$ with $n$ a positive integer: the first of these 2 equalities should instead read $k=-(n+2) / n$ yielding $k=-3,-2,-5 / 3,-3 / 2, \ldots$ (note that the values of $k$ yielded by the definition $k=-(2 n+1) /(2 n-1)$ with $n$ an arbitrary positive integer coincide with those yielded by the definition $k=-(m+2) / m$ only if $m$ is an odd positive integer $)$.

Let us conclude by expressing the wishful hope that the type of approach used in the present paper be also applicable to other systems of nonlinear ODEs or PDEs - possibly also with discrete rather than continuous independent variables.

\section{Acknowledgements}

It is a pleasure to thank our colleagues Robert Conte, François Leyvraz and Andrea Giansanti for very useful discussions. We also like to acknowledge with thanks 2 grants, facilitating our collaboration - mainly developed via e-mail exchanges - by making it possible for FP to visit twice the Department of Physics of the University of Rome "La Sapienza": one granted by that University, and one granted jointly by the Istituto Nazionale di Alta Matematica (INdAM) of that University and by the International Institute of Theoretical Physics (ICTP) in Trieste in the framework of the ICTP-INdAM "Research in Pairs" Programme. Finally, we also like to thank Fernanda Lupinacci who, in these difficult times - with extreme efficiency and kindness - facilitated all the arrangements necessary for the presence of FP with her family in Rome.

\section{References}

[1] F. Calogero, Isochronous systems, Oxford University Press, Oxford, UK, hardback 2008, paperback 2012 .

[2] F. Calogero, R. Conte and F. Leyvraz, "New algebraically solvable systems of two autonomous first-order ordinary differential equations with purely quadratic right-hand sides", J. Math. Phys. 61, 102704 (2020).

[3] F. Calogero and F. Payandeh, "Solution of the system of two coupled first-order ODEs with second-degree polynomial right-hand sides", Math. Phys., Analysis, Geom., (submitted to, 24.12.2020).

[4] F. Dumortier, J. Llibre and J. C Artés, Qualitative theory of planar differential systems. Springer, Berlin, 2006. 
[5] R. Garnier, "Sur des systèmes différentielles du second ordre dont l'intégrale générale est uniforme", C. R. Acad. Sci. Paris 249, 1982-1986 (1959) and Ann. École Norm. 77(2), 123-144 (1960). 